\title{
A short course of oral amiodarone improves sinus rhythm maintenance post-cardioversion for atrial fibrillation
}

\author{
C Boos, J Ritzema, R S More
}

Heart 2004;90:1063-1064. doi: 10.1136/hrt.2003.022665

D irect current electrical cardioversion (DEC) for persistent atrial fibrillation $(\mathrm{AF})$ carries a very high initial success rate of up to $95 \%$, but as few as $23 \%$ of patients remain in sinus rhythm one year post-procedure, with most recurrences of $\mathrm{AF}$ occurring within the first five days. ${ }^{1}$ Amiodarone has emerged as the most effective agent at preventing relapse of AF post-DEC, with up to $69 \%$ of patients remaining in sinus rhythm at one year. ${ }^{2}$ However, as many as $25 \%$ of amiodarone treated patients are forced to discontinue treatment because of side effects of the drug. ${ }^{3}$ Thus, increasing efforts have been made to improve the success rates for maintenance of sinus rhythm post-DEC, without exposing patients to the potentially toxic side effects of prolonged antiarrhythmic treatment. In this study we investigated whether a short four week course of oral amiodarone, pericardioversion for persistent AF, would improve medium and longer term sinus rhythm maintenance, without the significant drug side effects associated with prolonged amiodarone usage.

\section{METHODS}

The study recruited a cohort of 35 patients in persistent AF, $>1$ month duration without a reversible cause, referred for DEC. The patients were randomised (unblinded) into two groups. Group A received a total of four weeks treatment with oral amiodarone according to the following schedule: $200 \mathrm{mg}$ three times daily for one week before DEC, followed by $200 \mathrm{mg}$ three times daily during week 2, $200 \mathrm{mg}$ twice daily during week 3, then $200 \mathrm{mg}$ once a day for week 4 . Group B continued with the usual treatment but did not receive amiodarone or any other antiarrhythmic therapy.

The cardioversion protocol consisted of up to five synchronised monophasic shocks (delivered by Codemaster XL, Hewlett Packard), anterior-anterior $200 \mathrm{~J}, 360 \mathrm{~J}, 360 \mathrm{~J}$, then anterior-posterior $360 \mathrm{~J}$ and $360 \mathrm{~J}$. The cardioversion protocol was terminated by either technical success (defined in our case as sinus rhythm confirmed by a 12 lead ECG and maintained for at least 20 minutes after successful cardioversion) or the delivery of a sequence of five shocks. ${ }^{4}$

All patients were anticoagulated with warfarin (international normalised ratio (INR) of 2.0-3.0) for a minimum of four weeks before and four weeks after DEC. Exclusion criteria were as follows: patients who had undergone a DEC within the previous six months, patients $>80$ years or $<18$ years old, and patients with prosthetic valves or with significant heart failure (New York Heart Association stage III-IV). All patients had the following parameters measured before DEC: resting 12 lead ECG, renal serum, liver, and thyroid function tests, and transthoracic echocardiography. The INR was checked before inclusion, at five days post-commencement of amiodarone therapy, and at three weeks post-DEC.

Patients were assessed post-cardioversion by 12 lead ECG at six weeks and six months. The study was powered for the primary end point of sinus rhythm maintenance at six months. In addition, all patients were seen at a minimum of 12 months post-cardioversion and their sinus rhythm was assessed by 12 lead ECG. Secondary end points included total shock energy, number of shocks utilised for DEC, adverse drug effects related to amiodarone treatment, and six week sinus rhythm rates.

With a two sided $\alpha$ of 0.05 and power $80 \%, 17$ patients were needed per group assuming a six month sinus rhythm rate of $75 \%$ for amiodarone treated patients and 20\% for control patients. Comparisons between the two groups were made by Student's $t$ test, with differences in ratios assessed by Fisher's exact test. A probability value of $p<0.05$ was considered significant. Data are expressed as mean (SD).

\section{RESULTS}

The two groups were well matched for baseline characteristics. There were no significant differences between the groups for the following variables: age, weight, left ventricular ejection fraction, left atrial dimension, left ventricular dimensions in systole and diastole, mean duration of documented $\mathrm{AF}$, serum potassium concentration on day of cardioversion, mean ventricular rate pre-cardioversion, and AF risk factors (table 1). Furthermore, there was also no difference in the use of $\beta$ blockers, digoxin, and rate controlling calcium antagonists between the two groups. No patient withdrawals occurred from the study after randomisation. None of the recruited patients had spontaneously cardioverted before the planned DEC. There were no major adverse drug effects in the amiodarone group, with the exception of one patient, who missed a three week post-DEC appointment and was noted to have an uncomplicated prolongation of an INR of 6.1, at one month post-DEC.

The sinus rhythm maintenance rate was significantly higher in the amiodarone group at the six week (88\% $v$ $17 \%, \mathrm{p}<0.0001)$ and six month $(65 \% v 17 \%, \mathrm{p}=0.0016)$ follow up periods, compared with the control group. A nonsignificant trend to a higher rate of sinus rhythm in the amiodarone group was seen after 16.2 (4.1) months compared with the control group $(47 \% \vee 17 \%, \mathrm{p}=0.075)$.

\section{DISCUSSION}

This is the first clinical study with longer term outcome data to compare directly the effects of short term oral amiodarone treatment to facilitate DEC in patients with persistent AF. Short course oral amiodarone treatment proved superior to usual therapy in preventing AF relapse after successful DEC, although this effect lessened with progressive follow up (while $88 \%$ of amiodarone treated patients were in sinus rhythm six weeks after a successful DEC, this figure fell to $47 \%$ after 16 months). The efficacy of short course amiodarone treatment appears to be due to the prevention of early AF relapse commonly noted after DEC. These data support other recent studies that suggest the use of prolonged anticoagulation, even if sinus rhythm is initially achieved, as long term sinus rhythm maintenance cannot be guaranteed. ${ }^{1}$

Abbreviations: AF, atrial fibrillation; DEC, direct current electrical cardioversion; INR, international normalised ratio 
Table 1 Comparison of clinical characteristics and outcomes of amiodarone treated patients and control patients

\begin{tabular}{|c|c|c|c|}
\hline & $\begin{array}{l}\text { Amiodarone treated } \\
\text { patients (group A) }\end{array}$ & $\begin{array}{l}\text { Control patients } \\
\text { (group B) }\end{array}$ & p Value \\
\hline Number of patients & 17 & 18 & NS \\
\hline Male/female & $14 / 3$ & $15 / 3$ & NS \\
\hline Age (years) & $61.2(12.3)$ & $61.6(7.6)$ & NS \\
\hline Mean duration of documented AF (months) & $7.2(4.2)$ & $10.2(6.0)$ & NS \\
\hline Weight (kg) & $85.4(8)$ & $85.4(12.5)$ & NS \\
\hline Number of patients with $\geqslant 1$ previous DEC & 4 & 2 & NS \\
\hline Serum $\mathrm{K}^{+}$pre-cardioversion $(\mathrm{mmol} / \mathrm{l})$ & $4.6(0.5)$ & $4.4(0.5)$ & NS \\
\hline \multicolumn{4}{|l|}{ Number of patients with risk factors for AF } \\
\hline Hypertension & 11 & 11 & NS \\
\hline IHD & 6 & 3 & NS \\
\hline Lone & 2 & 2 & NS \\
\hline $\mathrm{DM}$ & 2 & 1 & NS \\
\hline Number of patients taking rate controlling agents & $16 / 17(94.1 \%)$ & $17 / 18(94.4 \%)$ & NS \\
\hline$\beta$ Blockers & $8 / 17$ & $9 / 18$ & NS \\
\hline Digoxin & $7 / 17$ & $5 / 18$ & NS \\
\hline Rate controlling calcium antagonist & $1 / 17$ & $3 / 17$ & NS \\
\hline EF & $50.2(13.5 \%)$ & $51(13.0 \%)$ & NS \\
\hline $\operatorname{LAD}(\mathrm{cm})$ & $4.4(0.6)$ & $4.1(0.8)$ & NS \\
\hline $\operatorname{LVEDD}(\mathrm{cm})$ & $4.8(0.4)$ & $4.6(0.5)$ & NS \\
\hline LVESD $(\mathrm{cm})$ & $3.4(0.5)$ & $3.4(0.7)$ & NS \\
\hline Ventricular rate pre-cardioversion (bpm) & $90.8(14.7)$ & $88.1(18.0)$ & NS \\
\hline Total shock energy used for cardioversion $(\mathrm{J})$ & $454(331)$ & $620(498)$ & NS \\
\hline Number of shocks utilised & $1.7(0.9)$ & $2.2(1.4)$ & NS \\
\hline Initial success rate for cardioversion & $17 / 17(100 \%)$ & $17 / 18(94 \%)$ & NS \\
\hline SR rate at 6 weeks & $15 / 17(88 \%)$ & $3 / 18(16.7 \%)$ & $<0.0001$ \\
\hline SR rate at 6 months & $11 / 17(65 \%)$ & $3 / 18(16.7 \%)$ & 0.0016 \\
\hline At 16 months follow up & $8 / 17(47.1 \%)$ & $3 / 18(16.7 \%)$ & 0.075 \\
\hline \multicolumn{4}{|c|}{$\begin{array}{l}\text { AF, atrial fibrillation; DM, diabetes mellitus; } E F \text {, ejection fraction; IHD, ischaemic heart disease; } \mathrm{K}^{+} \text {, potassium } \\
\text { LAD, left atrial dimension; LVEDD, left ventricular end diastolic dimension; LVESD, left ventricular end systolic } \\
\text { dimension; NS, not significant; sinus rhythm, sinus rhythm. } \\
\text { Data are expressed as mean (SD). }\end{array}$} \\
\hline
\end{tabular}

Recent trials have highlighted increased rates of hospitalisation and adverse effects seen with antiarrhythmic therapy. ${ }^{13}$ It is thus encouraging that short course treatment with amiodarone is able to improve cardioversion results without exposing patients to the long term adverse effects of antiarrhythmic therapy, although INR levels must be very closely monitored.

The amiodarone dosage schedule used in this study is an adaptation of a commonly used dosage regimen. Although it can take several weeks to achieve steady state serum amiodarone concentrations, even with a loading regimen, adequate amiodarone concentrations can be achieved quickly in the majority of patients when even a single loading dose is used. ${ }^{5}$ We used a treatment schedule that would minimise the length of time our patients were exposed to amiodarone, yet hopefully ensured adequate amiodarone concentrations in the pericardioversion period.

This trial has several limitations. The study was unblinded and not placebo controlled, although hard objective clinical end points were used. Secondly, this was a small study of only 35 patients which was adequately powered for six month follow up but not for longer follow ups. Thirdly, the rate of sinus rhythm maintenance in the group not treated with amiodarone was low at $16.6 \%$ after six months. This is lower than some rates quoted in the literature, but is comparable to historical rates we had observed from prior auditing and follow up of patients attending our cardioversion clinic (this presumably relates to our patient case mix including their duration of $\mathrm{AF}$ before cardioversion). Furthermore, other authors have also quoted low rates of maintenance of sinus rhythm. ${ }^{6}$ Finally, follow up assessment of liver and thyroid function and subclinical AF burden (by Holter monitoring) would have been preferable but were not undertaken in this study.

For patients in persistent AF, short burst therapy with oral amiodarone appears to significantly improve six week and six month sinus rhythm maintenance rates post-DEC, without exposing patients to the side effects of long term amiodarone treatment. Larger randomised studies are required to determine whether pericardioversion use of oral amiodarone is as effective as long term amiodarone treatment in maintaining sinus rhythm after successful cardioversion.

\section{ACKNOWLEDGEMENTS}

The authors would like to thank Kay Hughes, Joy Baker, Roger Hoke, and the nurses of our cardiac unit for their technical support. We would also like to thank Dr Matthew Dawes for his help with the preparation of this manuscript.

\section{Authors' affiliations}

C Boos, J Ritzema, Department of Cardiology, St Mary's Hospital, Portsmouth, UK

R S More, Department of Cardiology, Victoria Hospital, Blackpool, UK

Correspondence to: Dr Christopher J Boos, 18C Nightingale Road, Southsea, Portsmouth, Hampshire, PO5 3JL, UK; christopherboos@ hotmail.com

Accepted 27 November 2003

\section{REFERENCES}

1 Boos C, More RS, Carlsson J. Persistent atrial fibrillation: rate control or rhythm control. BMJ 2003;326:1411-2.

2 Roy D, Talajic M, Dorian $P$, et al. Amiodarone to prevent recurrence of atrial fibrillation. Canadian trial of atrial fibrillation investigators. N Engl J Med 2000;342:913-20.

3 Hohnloser SH, Kuck KH, Lilienthal J. Rhythm or rate control in atrial fibrillation-pharmacological intervention in atrial fibrillation (PIAF): a randomised trial. Lancet 2000;356:1789-94.

4 Boos C, Thomas M, Jones A, et al. Higher energy monophasic DC cardioversion for persistent atrial fibrillation: is it time to start at 360 joules? Ann Noninvasive Electrocardiol 2003;8:121-6.

5 Nielsen KD, Moller S. Amiodarone for rapid cardioversion of chronic atrial tachyarrhythmia? Pharmacol Toxicol 2000;86:283-6.

6 Lundstrom T, Ryden L. Chronic atrial fibrillation: long term results of direct current cardioversrion. Acta Med Scand 1988;223:53-9. 